\title{
Finding heavy long-lived charged particles with the ATLAS detector
}

\author{
Sofia Vallecorsa * \\ For the ATLAS Collaboration
}

\begin{abstract}
Long-lived charged particles are predicted by many models of physics beyond the Standard Model (SM). At the LHC, the common signature is a heavy long-lived charged particle with velocity smaller than the speed of light, $\beta<1$. This paper presents methods we developed for identifying slow particles and measuring their mass using the ATLAS muon spectrometer. The efficacy of these methods is demonstrated using two different models.
\end{abstract}

\section{Heavy particle signature in ATLAS}

ATLAS was designed to fully exploit the LHC discovery potential [1] [2] but the scenario of slowly moving particles was not considered. With $\beta<1$, a particle may be lost during data collection. We offer a method to identify such particles and measure

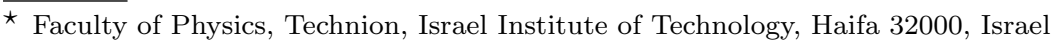

Preprint submitted to Elsevier

4 February 2009 
their mass [3] and demonstrate its efficacy using two different models: Gauge Mediated SUSY Breaking point 5 (GMSB5) [4], and split SUSY R-Hadrons (hadronized gluinos) [5] with a mass of $300 \mathrm{GeV}$. These methods ensure the acceptance and identification of these particles from the data acquisition, through the trigger, and in event reconstruction. A long-lived charged slepton interacts in the detector in a way similar to a muon. Thus the least model dependent signature is a charged particle with low $\beta$ which reaches the muon chambers and the largest background is high $p_{T}$ muons with mis-measured timing. Since ATLAS is so large that information from 3 separate bunch crossings (BC) co-exists at the same time, it is crucial to match correctly event fragments from different subdetectors [6]: when $\beta$ is small, the particle will take longer to reach the detectors and hits may not be read-out. In order to find all hits from slow particles ATLAS must collect hits from the consecutive BC.

\section{Triggering on long-lived heavy charged particles}

ATLAS has a three-level trigger system [1].

- At the first level (L1) [6], a long-lived charged particle is most likely to trigger as a muon. The L1 muon trigger, based on dedicated fast detectors (RPCs and TGCs), identifies the correct detector region and $\mathrm{BC}$.

- The second level trigger (L2) [7] analyzes data acquired by L1 and includes two software stages. For muons, the first stage reconstructs the muon $p_{T}$ in the spectrometer. The second matches an inner detector track with the muon spectrometer track and refines the $p_{T}$ estimate. We developed an algorithm to trigger on heavy stable charged particles, by measuring their time of flight and velocity. It uses the excellent time resolution (3ns) of the RPC chambers located in the barrel of the muon spectrometer. Our selection requires $p_{T}>40 \mathrm{GeV}, \beta<0.97$ and $m>40 \mathrm{GeV}$. The efficiency is above $90 \%$ for GMSB sleptons and R-Hadrons events in the barrel. Figure 1 (left) shows the mass distribution of GMSB5 signal and background. The $p_{T}$ resolution is improved by using a matching inner detector track, if it exists (right). If the R-Hadron is identified as a slow particle candidate, it is accepted without the matching inner detector track, to prevent the loss of R-Hadrons that are neutral in the inner detector or have inner detector hits in the previous BC.

- The final trigger decision is made in the event filter (EF) [7], which uses algorithms adapted from the offline reconstruction. Below we present a specific reconstruction algorithm which includes identification of charge-flipped (or late) R-Hadrons (no inner detector track). The identification based only on the muon spectrometer is applied in two cases; if the particle was already identified by the L2 stau selection in the barrel, or for high $p_{T}$ muon candidates in the end-cap that do not have an inner detector track. Standard muon trigger and the slow particle trigger are compared in table 1.

\section{Identifying heavy long-lived particles at reconstruction}

In ATLAS, muons are reconstructed using the MDTs, precision muon chambers, in combination with the RPC, TGC and CSC sub-detectors [1]. Standard reconstruction efficiency starts dropping sharply for $\beta<0.75$ and goes to 0 at $\beta=0.4$. This is due to two main issues: the data may not be collected if the particle hits are in the next $\mathrm{BC}$; late 

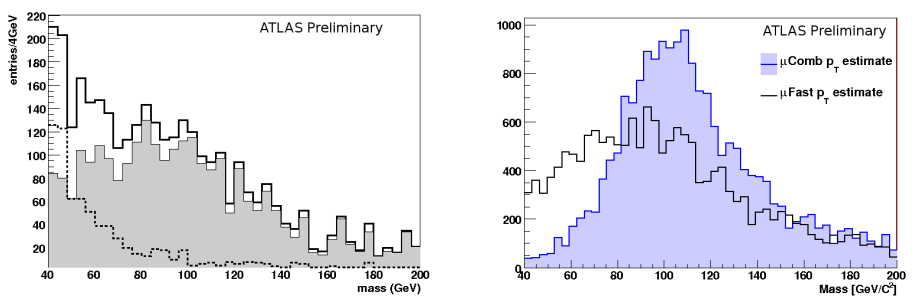

Fig. 1. Mass distribution of signal and background resulting from the L2 selection for an integrated luminosity of $100 \mathrm{pb}-1$ (left). The shaded area is the signal, the dashed line is the muon background, and the full line is the sum. The shaded area in the plot on the right shows the mass resolution when a matching inner detector track is found.

\section{Table 1}

\begin{tabular}{|l|c|c|}
\hline Trigger algorithm & R-Hardons 300 GeV & Sleptons $\beta=0.6$ in barrel \\
\hline \hline L2 MS & $98 \%(98 \%)$ & $99 \%(100 \%)$ \\
L2 MS+ID & $98 \%(49 \%)$ & $99 \%(100 \%)$ \\
EF & $72 \%(23 \%)$ & $97 \%(39 \%)$ \\
\hline
\end{tabular}

Slow particle trigger efficiencies for heavy stable charged particles, with respect to L1. The numbers in parentheses refer to efficiencies for the stadard muon trigger.

arrival of the slepton disrupts segment fitting in the MDTs. We reconstruct slow particles and estimate their mass with a muon identification package [8] which starts from inner detector tracks and looks for corresponding hits in the muon spectrometer, identifing candidates even when the segment reconstruction is imperfect. It is based on 3 techniques: recovering trigger detector hits from the next $\mathrm{BC}$, estimating the particle velocity from the RPC hit time and by selecting the $\beta$ that minimizes the MDT segments $\chi^{2}$. The mass resolution can be further improved when the full track is re-fit. The algorithm yields a high efficiency even for low values of $\beta$ (greater than $90 \%$ for $\beta>0.4$ ).

\section{Conclusions}

Heavy long-lived charged particles can be discovered in ATLAS. However this must be done in the data acquisition, high level trigger and reconstruction stages and it cannot be done with the standard ATLAS tools. We have presented a model independent method to measure their mass in the trigger and reconstruction software.

\section{References}

[1] G. Aad et al., ATLAS Collaboration, JINST 3 (2008) S008003.

[2] ATLAS Collaboration, CERN-OPEN-2008-020, 2008

[3] S. Tarem, S.Bressler, H. Nomoto, A. Di Mattia, SN-ATLAS-2008-071, 2008.

[4] G.F. Giudice, R. Rattazzi, Phys. Rep. 322 (1999) 41999.

[5] N. Arkani-Hamed, S. Dimopoulos, J. High Energy Phys.0506 (2005) 073.

[6] ATLAS Level-1 Trigger Group, ATLAS TDR 12, Aug. 1998.

[7] ATLAS HLT/DAQ/DCS Group, ATLAS TDR 16, Nov. 2002.

[8] S. Tarem, Z. Tarem, N. Panikashvili, O. Belkind, Nuc. Sc. Symposium, 2006. IEEE Vol. 1, May 2007, 617-621. 